\title{
Musical Training Shapes Structural Brain Development
}

\author{
Krista L. Hyde, ${ }^{1}$ Jason Lerch, ${ }^{2}$ Andrea Norton, ${ }^{4}$ Marie Forgeard, ${ }^{4}$ Ellen Winner, ${ }^{3}$ Alan C. Evans, ${ }^{1}$ and Gottfried Schlaug ${ }^{4}$ \\ ${ }^{1}$ McConnell Brain Imaging Center, Montreal Neurological Institute, McGill University, Montreal, Quebec, Canada H3A 2B4, ${ }^{2}$ Mouse Imaging Centre, \\ Hospital for Sick Children, Toronto, Ontario, Canada M5T 3H7, ${ }^{3}$ Department of Psychology, Boston College, Chestnut Hill, Massachusetts 02467, and \\ ${ }^{4}$ Music and Neuroimaging Laboratory, Department of Neurology, Beth Israel Deaconess Medical Center and Harvard Medical School, Boston, \\ Massachusetts 02215
}

The human brain has the remarkable capacity to alter in response to environmental demands. Training-induced structural brain changes have been demonstrated in the healthy adult human brain. However, no study has yet directly related structural brain changes to behavioral changes in the developing brain, addressing the question of whether structural brain differences seen in adults (comparing experts with matched controls) are a product of "nature" (via biological brain predispositions) or "nurture" (via early training). Longterm instrumental music training is an intense, multisensory, and motor experience and offers an ideal opportunity to study structural brain plasticity in the developing brain in correlation with behavioral changes induced by training. Here we demonstrate structural brain changes after only 15 months of musical training in early childhood, which were correlated with improvements in musically relevant motor and auditory skills. These findings shed light on brain plasticity and suggest that structural brain differences in adult experts (whether musicians or experts in other areas) are likely due to training-induced brain plasticity.

\section{Introduction}

Studies comparing adult musicians with matched nonmusicians have revealed structural and functional differences in musically relevant brain regions such as sensorimotor brain areas (Elbert et al., 1995; Hund-Georgiadis and von Cramon, 1999; Schlaug, 2001; Gaser and Schlaug, 2003b), auditory areas (Pantev et al., 1998; Zatorre, 1998; Schneider et al., 2002; Gaab and Schlaug, 2003; Bermudez and Zatorre, 2005; Lappe et al., 2008), and multimodal integration areas (Münte et al., 2001; Sluming et al., 2002, 2007; Gaser and Schlaug, 2003a; Lotze et al., 2003; Bangert and Schlaug, 2006; Zatorre et al., 2007). While some research has investigated functional brain correlates of musical training in childhood (Overy et al., 2004; Koelsch et al., 2005; Fujioka et al., 2006; Shahin et al., 2008), no studies have yet examined structural brain and behavioral changes in the developing brain in response to long-term music training to specifically address the question of whether structural brain differences seen in adults (comparing experts with matched controls) are a product of "nature" or "nurture."

Such a study could also examine cognitive and behavioral changes in parallel with brain changes in response to music training. There is a widespread view that learning to play a musical instrument in childhood stimulates cognitive development and leads to the enhancement of skills in a variety of extramusical

Received 0ct. 23, 2008; revised Jan. 6, 2009; accepted Jan. 27, 2009.

This work was supported by grants from the National Science Foundation (BCS0518837), the Dana Foundation, and the NAMM Foundation. We thank our previous research assistants and postdoctoral fellows (K. Cronin, L. Forbes, L. Blake, C. Alexander, M. Rosam, K. Brumm, A. Norton, L. Zhu, U. lyengar, and K. Overy) for test preparation, behavioral testing, and imaging data collection and the participating children and their families for their cooperation in taking part in our experiments.

Correspondence should be addressed to either Krista L. Hyde or Gottfried Schlaug at the above addresses. E-mail: krista.hyde@mail.mcgill.ca or gschlaug@bidmc.harvard.edu.

DOI:10.1523/JNEUROSCI.5118-08.2009

Copyright $\odot 2009$ Society for Neuroscience $\quad$ 0270-6474/09/293019-07\$15.00/0 areas, which is commonly referred to as transfer (Bangerter and Heath, 2004). The most commonly observed form of transfer occurs when there is a close resemblance between the training domain and the transfer domain (typically referred to as "near transfer," e.g., fine motor skills that develop while learning to play a musical instrument lead to increased speed and accuracy in typing). While near-transfer effects are relatively common, it is notoriously difficult to demonstrate "far transfer," where the resemblance between training and transfer domains is much less obvious (e.g., learning to read and perform with precision from musical rhythm notation and understanding fractions in math). There are some claims for far transfer from instrumental music training in the areas of verbal, spatial, mathematical, and intelligence quotient (IQ) performance (Rauscher et al., 1993, 1997, 1998; Chan et al., 1998; Ho et al., 2003; Schellenberg, 2004; Forgeard et al., 2008), but such findings have also been controversial (Steele et al., 1999).

As part of an ongoing longitudinal study on the effects of music training on brain, behavioral, and cognitive development in young children (Norton et al., 2005; Schlaug et al., 2005), here we investigated structural brain changes in relation to behavioral changes in young children who received 15 months of instrumental musical training relative to a group of children who did not. We used deformation-based morphometry (DBM), an unbiased and automated approach to brain morphology, to search throughout the whole brain on a voxelwise basis for local brain size or shape differences between groups (Collins et al., 1994; Robbins et al., 2004). The DBM technique is useful for measuring morphometric brain changes longitudinally, as in the present study, where the DBM metric of interest, the Jacobian determinant, yields a measure of relative voxel size change over time in terms of voxel expansion (growth) or contraction (shrinkage). To investigate a brain-behavioral relationship, we correlated the 
Table 1. Subject characteristics

\begin{tabular}{lcc}
\hline Characteristics & Instrumentals $(n=15)$ & Controls $(n=16)$ \\
\hline Age at start of study (SD) & $6.32(0.82)$ years & $5.90(0.54)$ years \\
Time from MRI scan 1 to scan 2 (SD) & $15.60(3.30)$ months & $14.80(3.80)$ months \\
Socioeconomic standard* & $5.10(0.60)$ & $4.60(0.80)$ \\
Gender & 9 females; 6 males & 7 females; 9 males \\
\hline
\end{tabular}

* Socioeconomic standard was defined on a six-point scale, with a score of 1 reflecting that the children's parents had some high school education, to a score of 6, reflecting that parents had a doctoral degree (Norton et al., 2005).

brain deformation changes after 15 months with performance changes on behavioral tests.

\section{Materials and Methods}

Participants. We tested two groups of children that were recruited from Boston area public schools and who had no prior formal musical training (see Table 1). The "instrumental" group consisted of 15 children (mean age at start of study 6.32 years old, SD 0.82 years) beginning weekly half-hour private keyboard lessons (outside of the school system), and who continued lessons for a mean interval of 15 months. The "control" group consisted of 16 children (mean age at start of study 5.90 years old, SD 0.54 years) who did not receive any instrumental music training during this 15 month period, but did participate in a weekly $40 \mathrm{~min}$ group music class in school consisting of singing and playing with drums and bells. The instrumental and control children were all right handed and matched as closely as possible in gender, age at the start of the study, and socioeconomic status (SES). SES was defined by parental education on a six-point scale, with a score of 1 , for children whose parents had some high school education, to a score of 6 , for those whose parents had a doctoral degree (see Norton et al., 2005).

At time 1, all children were tested on a series of behavioral tests (described below), and underwent a magnetic resonance imaging (MRI) scan (scan 1). At time 2 (15 months later), all children were retested on the behavioral tests and underwent a second MRI scan (scan 2). The children whose results are reported here are drawn from a slightly larger group of instrumental and control children (see Norton et al., 2005). Here we only report the results from the children who completed both the behavioral tests and MRI scanning at times 1 and 2. We tested the hypothesis that brain and behavioral changes after 15 months should be greater in instrumental than in control children; this time period allows us to compare our results with those of other studies using a similar observation period.

Behavioral tests and MRI scanning. Children were tested individually at times 1 and 2 on measures of handedness and SES, and on two neartransfer measures: a four-finger motor sequencing test for the left and right hands assessing fine finger motor skills, and a custom-made "melodic and rhythmic discrimination test battery" assessing music listening and discrimination skills. Five far-transfer measures were also administered: the object assembly, block design, and vocabulary subtests of the WISC-III (Wechsler, 1991), the Raven's progressive matrices (colored progressive matrices and standard progressive matrices) (Raven, 1976a,b), and the auditory analysis test (Rosner and Simon, 1971), assessing phonemic awareness. The vocabulary subtest of the WISC was used as a proxy for verbal IQ. For a detailed description of these tests and their administration to this group of children, see Norton et al. (2005) and Forgeard et al. (2008).

The two musically relevant (near transfer) behavioral tests are described in more detail below, since these were the only tests that showed significant between group differences after 15 months (see below, Results). Both of these tests are related to musical activity, but can also be performed by children who do not have any instrumental music training. In the four-finger motor sequencing test, children pressed a particular number sequence (e.g., 5-2-4-3-5) corresponding to fingers 2-5 of their left or right hand on the number keys of a computer keyboard as often, accurately, and fast as possible over a 30 s period. In the "melodic and rhythmic discrimination test battery," children heard pairs of five-tone musical phrases differing only in melody and pairs of phrases differing only in rhythm. The task was to indicate whether the two musical phrases were the same or different. These musical phrases were designed for this study and have been described in more detail previously (Overy et al., 2004; Norton et al., 2005; Forgeard et al., 2008). The melodic and rhythmic subtest scores were combined to form one single behavioral measure of auditory-musical discrimination. Behavioral "difference scores" measuring the difference in performance on the behavioral tests from time 1 to time 2 were calculated and then correlated with the brain deformation measures.

Anatomical MRI scans were obtained for all children on a 3T General Electric MRI scanner using a T1-weighted, magnetization-prepared gradient-echo volume acquisition with a voxel resolution of $0.93 \times$ $0.93 \times 1.5 \mathrm{~mm}$. This research was approved by the ethics committees of the Beth Israel Deaconess Medical Center. Written informed consent was obtained from the parents of all the children, and the children themselves gave assent to participate in this study.

Brain deformation-based morphometry analyses. Automated deformation brain analyses were performed on the T1 MRI data for each child (see supplemental Fig. 1, available at www.jneurosci.org as supplemental material). All MRI scans were first nonuniformity corrected (Sled et al., 1998), and registered to MNI space with a nine-parameter linear transform using mni_autoreg tools (Collins et al., 1994; Robbins et al., 2004). Next, brain deformation measures in terms of the Jacobian determinants (yielding a measure of relative voxel expansion or contraction) were calculated so that we could perform three different statistical analyses. First, to test for any brain deformation differences at baseline (before musical training), for each group, all time 1 MRI scans (0 months) iteratively underwent nonlinear registration toward the previous group average (starting with the linear group average). The Jacobian determinants of the final nonlinear registration were computed and blurred with a 10 $\mathrm{mm}$ Gaussian kernel. Statistical analyses were then performed comparing the Jacobian determinant data between groups at baseline, at each voxel. Second, to test for brain deformation differences between groups over time, each child's time 1 scan (at 0 months) was nonlinearly aligned to his or her time 2 scan (15 months later). The resulting displacement field was blurred with a $10 \mathrm{~mm}$ Gaussian kernel and the Jacobian determinant of the blurred displacement field was computed. Statistical analyses were then performed comparing the longitudinal Jacobian determinant data between groups, at each voxel. Third, to test for a brainbehavioral relationship, brain deformation differences (Jacobian determinants of scan 2 - scan 1 as above) were regressed on the behavioral difference scores (difference in test performance time 1 to time 2), for each subject, at each voxel. Last we checked for T1-weighted intensity differences between groups. All scans were intensity normalized, each subject's time 1 scan was subtracted from their time 2 scan, and the resulting intensity differences were compared between groups in a linear model.

The general linear model was used in the group statistical analyses with age at time 1, gender, and SES entered as covariates. The results from the group comparison were thresholded using random field theory cluster thresholding (Friston et al., 1994; Worsley et al., 2004), with a $p<0.05$ cluster corresponding to at least 904 connected voxels with an uncorrected $p<0.001$, or at an a priori cluster threshold of $p<0.1$ (at least 240 connected voxels at an uncorrected $p<0.001$ ) for strongly predicted regions that were not significant at the whole-brain threshold. The significant brain deformation differences from the group comparison were then used to define a volume of interest in which to test for brainbehavior correlations with the scores on the motor and auditory-musical tests. The results from this volume of interest were thresholded using the false discovery rate theory (Genovese et al., 2002) at $q=0.05$.

\section{Results}

\section{Behavioral changes}

An initial $\chi^{2}$ analysis showed no significant difference between the instrumental and control groups in gender distribution $(p>$ 0.1 ). Initial ANOVAs showed no significant difference between the groups in vocabulary scores at baseline $(p>0.1)$, replicating the results initially reported in Norton et al. (2005). There was a significant difference between groups in SES, with the instrumen- 
tal group (mean 5.1 points, SD 0.63) having a higher average SES than the control group (mean 4.47 points, SD 0.87 ). The two groups also differed slightly in age at baseline (time 1), with the instrumental group (mean 6.32 years, SD 0.82 ) $\sim 5$ months older than the control group (mean 5.90 years, SD 0.54). Although this age difference only approached significance $(p=0.1)$, we chose to be conservative and covaried age along with SES in our subsequent analyses.

A multiple analysis of covariance (MANCOVA), covarying age and SES, was conducted to determine that there were no preexisting group differences at time 1 on either near- or fartransfer outcomes. Missing values were replaced by the series' mean $(2.42 \%$ of all values). The MANCOVA revealed no significant overall difference between groups (Wilks' $\lambda=0.85, F_{(8,20)}$ $=0.44, p=0.88$ ). Follow-up univariate tests also indicated that the two groups did not differ significantly on any of the outcomes (all $p>0.1$ ). Furthermore, the groups did not differ significantly in interval length (in months) between baseline (time 1) and time 2 testing $(p>0.1)$

To determine whether the instrumental group progressed more than the control group on any of the outcomes between times 1 and 2, another MANCOVA was performed using the behavioral difference scores (performance difference from time 1 to 2) as our dependent variable, and age at baseline and SES as our covariates. Missing values were replaced by the series' mean (for $6.85 \%$ of all values). As predicted, there was a significant overall difference in the behavioral difference scores between the two groups (Wilks' $\lambda=0.50, F_{(8,20)}=2.55, p=0.04$, partial $\eta^{2}=$ $0.51)$. Univariate tests revealed differences in the two neartransfer outcomes (motor and melody/rhythm tests) but not in any far-transfer outcomes.

On the finger motor sequencing test, the instrumental group significantly outperformed the control group in terms of the right-hand motor performance improvement over time $\left(F_{(1,27)}\right.$ $=7.25, p=0.01$, partial $\left.\eta^{2}=0.21\right)$, and the difference between groups approached significance for the left hand $\left(F_{(1,27)}=3.81\right.$, $p=0.06$, partial $\left.\eta^{2}=0.12\right)$. The instrumental group also significantly outperformed the control group in improvement on the custom-made melodic/rhythmic discrimination test battery $\left(F_{(1,27)}=13.20, p<0.01\right.$, partial $\left.\eta^{2}=0.33\right)$. No between-group differences in improvement over time (time 1 to 2 ) were found for the far-transfer measures of block design, vocabulary, object assembly, Raven's progressive matrices, and auditory analysis (all $p>0.1)$.

\section{Brain deformation changes}

With regard to between-group brain differences, we did not see any differences between groups at time 1 . In terms of brain deformation changes in typical development that occurred in our controls $(n=15)$ over the 15 month period, brain deformations were found in frontal, temporal, and parieto-occipital brain areas (supplemental Fig. 2, available at www.jneurosci.org as supplemental material). In terms of between-group differences between the two time points, instrumental children showed significantly different brain deformation changes over the 15 months (time 2 scan at 15 months minus time 1 scan at 0 months) compared with controls (see Table 2 for all significant results). Instrumental children showed areas of greater relative voxel size than those of controls in motor areas, such as the right precentral gyrus (motor hand area) (Fig. 1a), and the corpus callosum (fourth and fifth segment/midbody) (Fig. 2a), that were significant at a wholebrain cluster threshold at $p<0.05$, as well as in a right primary auditory region (lateral aspect of Heschl's gyrus) (Fig. $3 a$ ) that
Table 2. Significant between-group longitudinal brain deformation differences

\begin{tabular}{llll}
\hline Brain area & $\begin{array}{l}\text { Number of } \\
\text { voxels in cluster }\end{array}$ & $\begin{array}{l}\text { RFT cluster } \\
p \text { value }\end{array}$ & $\begin{array}{l}\text { Brodmann } \\
\text { area }\end{array}$ \\
\hline
\end{tabular}

Relative voxel size increases

Left middle frontal gyrus
Corpus callosum

Left superior frontal gyrus

4744

3145

Right middle frontal gyrus

Left pericingulate

Right superior frontal gyrus

Left superior frontal gyrus

Right primary motor (precentral

gyrus)

Bilateral medial frontal gyrus

Right middle frontal gyrus

Right primary auditory (Heschl's gyrus)

Relative voxel size decreases

Left middle occipital gyrus

$\begin{array}{lr}0.0000 & \\ 0.0001 & 6 \\ 0.0011 & 8 \\ 0.0012 & 10 \\ 0.0014 & 31 \\ 0.0057 & 10 \\ 0.0097 & 9 \\ 0.0152,{ }^{*} 0.0014 & 6 \\ & \\ 0.0170 & 10 \\ 0.0434 & 11 \\ 0.5458,{ }^{*} 0.0717 & 41\end{array}$

$0.5458, * 0.0717 \quad 41$
All results are significant with whole-brain random field theory (RFT) cluster thresholding at $p<0.05$, with the exception of results with *, which are significant at an a priori cluster threshold of $p<0.1$ for strongly predicted regions.

was significant at an a priori cluster threshold at $p<0.1$. Some significant brain deformation differences were also found outside auditory and motor brain areas. Instrumental children showed areas of greater relative voxel size than those of controls in bilateral frontolateral and frontomesial regions and a left posterior pericingulate region. In comparison, instrumental children showed only one area of lesser relative voxel size than that of controls in the left middle occipital gyrus. Last, no differences in normalized MR intensities were found between the two groups.

\section{Correlations between brain and behavioral changes}

Brain deformation changes in motor-related brain areas, including the right precentral gyrus and the corpus callosum, were predicted by left-hand motor test improvement scores. To illustrate the relationship between brain morphometry and behavior, we plotted the longitudinal brain deformation change over 15 months (in terms of relative voxel size) for each child as a function of his or her behavioral difference score on the left-hand motor sequencing test at the most significant (peak) voxel in the right precentral gyrus and the corpus callosum. The relative voxel size significantly increased with increasing left-hand motor improvement score at peak voxels in the right precentral gyrus (Fig. $1 b$ ) and the corpus callosum (Fig. $2 b$ ), but not in the right primary auditory region. Brain deformation changes in the right auditory area (Fig. $3 b$ ) were predicted by improvements on the melodic/rhythmic discrimination test. However, brain deformation changes in the right primary motor region were not predicted by improvements on the melodic/rhythmic discrimination test battery, and brain deformation changes in the right primary auditory region were not predicted by motor improvement scores. No other significant correlations were found between brain deformations and either near or far-transfer behavioral measures.

\section{Discussion}

In the present study, we demonstrate regional structural brain plasticity in the developing brain that occurred with only 15 months of instrumental musical training in early childhood. Structural brain changes in motor and auditory areas (of critical importance for instrumental music training) were correlated with behavioral improvements on motor and auditory-musical 
tests. This study is the first longitudinal investigation to directly correlate brain structure and behavioral changes over time in the developing brain.

The lack of brain and behavioral differences between the instrumental and control children at baseline (before any music training) is consistent with previous findings from a larger sample that included the present subset of children tested here (Norton et al., 2005). It is not possible from these findings to completely rule out that musicians may be born with preexisting biological predictors of musicality or that some children may have a certain genetically determined trajectory of cerebral development that may lead them to more likely continue to practice music relative to other children without this same predisposition. However, our findings do support the view that brain differences seen in adult musicians relative to nonmusicians are more likely to be the product of intensive music training (Norton et al., 2005; Schlaug et al., 2005). Children who played and practiced a musical instrument showed greater improvements in motor ability (as measured by finger dexterity in both left and right hands) and in auditory melodic and rhythmic discrimination skills. Contrary to previous findings, however (Chan et al., 1998; Vaughn, 2000; Ho et al., 2003; Schellenberg, 2004; Rauscher et al., 1997, 2000), children who studied an instrument for 15 months did not show superior progress in visual-spatial and verbal transfer domain outcomes than children who did not receive instrumental training. We propose three reasons why 15 months of instrumental music training may not have been sufficient to result in far transfer: (1) 15 months of instrumental lessons may be too short a period of time (duration explanation); (2) children in our instrumental group may have practiced too little (intensity explanation); or (3) a larger sample may be required to demonstrate far transfer (power explanation).

The brain deformations found over 15 months in our controls (see supplemental Fig. 2, available at www.jneurosci.org as supplemental material) are consistent with previous findings in normal development that have included similar age ranges (from 5 to 7 years old) (e.g., Sowell et al., 2004). The consistency of the brain deformation found here in our controls with other studies of typical brain development in frontal, temporal, and parietooccipital brain areas strengthens our conclusions that the brain deformations observed here between instrumental and control children are due to musical training. The present findings of structural brain changes in response to 15 months of instrumental music training are consistent with previous findings of training-induced structural brain differences in adults in various contexts (Draganski et al., 2004; Draganski and May, 2008). More specifically, the brain deformation differences found in primary motor brain regions are consistent with structural brain differences found between adult musicians and nonmusicians in the precentral gyri (Gaser and Schlaug, 2003b) and the corpus callosum (Schlaug et al., 1995; Oztürk et al., 2002; Schmithorst and

\section{Primary motor area} (Instrument > Controls) Right precentral gyrus
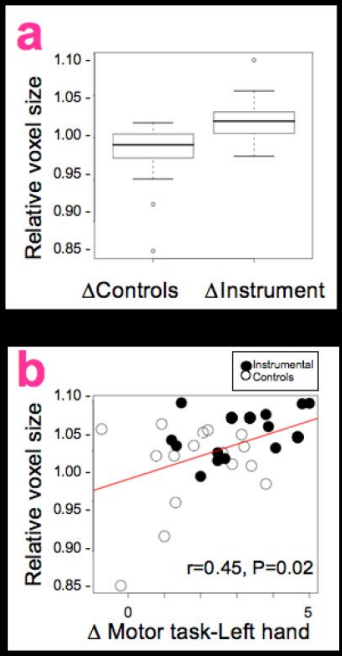

$\Delta$ Motor task-Left hand

Figure 1. Longitudinal group brain deformation differences and brain-behavioral correlations in primary motor area. The bottom lines of the box, SDs by the errors bars, and outliers by circles) is plotted for each group at the most significant (peak) voxe

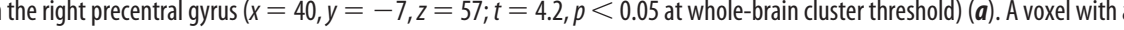
cores (from time 1 to time 2) of each child on the left-hand motor test that was found at the peak voxel in the right precentral

Wilke, 2002; Lee et al., 2003). Although the right auditory cluster was not significant at a whole-brain level, this result was strongly predicted on the basis of findings of previous structural brain differences in right auditory cortex in adult musicians (Schneider et al., 2002; Gaser and Schlaug, 2003b; Bermudez and Zatorre, 2005). Thus, we report this right primary auditory region at an a priori threshold.

The brain-behavioral correlations found here in motor and auditory brain regions for performance on motor and auditory (melodic/rhythmic) tests show that different motor and auditory behavioral functions (both musically relevant) appear to be driving the group differences in separate predicted brain regions. These results are important from a functional perspective since these brain regions are known to be of critical importance in instrumental music performance and auditory processing. For example, the primary motor area plays a critical role in motor planning, execution, and control of bimanual sequential finger movements as well as motor learning (Karni et al., 1995; Grodd et al., 2001). The correlation found between the brain deformation measures and the motor test at the corpus callosum is consistent with the fact that the peak voxel lies in the fourth and fifth segments of the corpus callosum (Witelson, 1989) (also called midbody), which contains fibers connecting primary sensorimotor cortex (Wahl et al., 2007). Moreover, it has been suggested that intense bimanual motor training of musicians could play an important role in the determination of callosal fiber composition and size (Schlaug et al., 1995). Last, the correlation found be- 


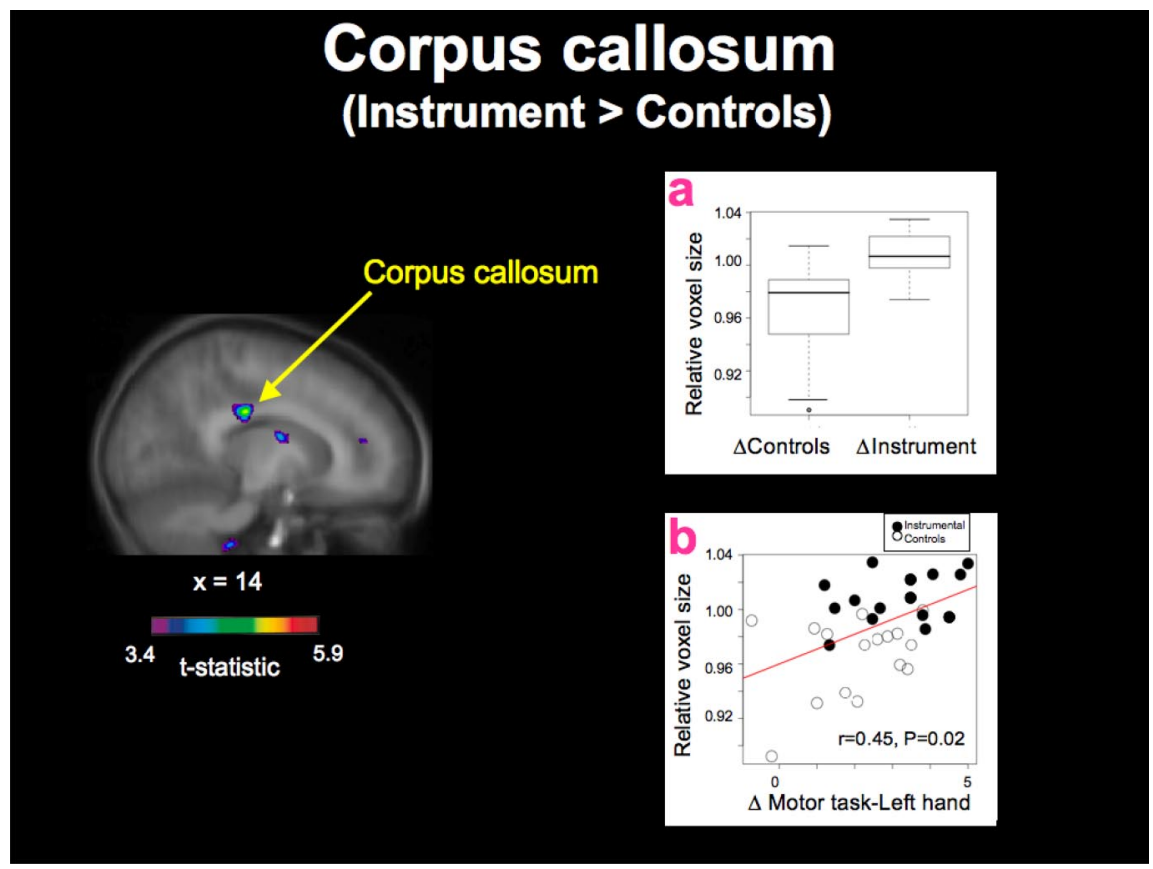

Figure 2. Longitudinal group brain deformation differences and brain-behavioral correlations in the corpus callosum. The brain image (a sagittal slice) shows areas of significant difference in relative voxel size over 15 months in instrumental $(n=15)$ versus control $(n=16)$ children in terms of a $t$-statistical color map of the significant clusters superimposed on an average MR image of all children $(n=31)$. The yellow arrow points to the corpus callosum. To illustrate the group differences, the relative voxel size is plotted for each group at the most significant (peak) voxel in the corpus callosum ( $x=14, y=-24, z=30 ; t=5.2$, $p<0.05$ at whole-brain cluster threshold) (a). The significant positive correlation of relative voxel size with behavioral difference scores (from time 1 to time 2) of each child is shown for the left-hand motor test at the peak voxel in the corpus callosum (b).

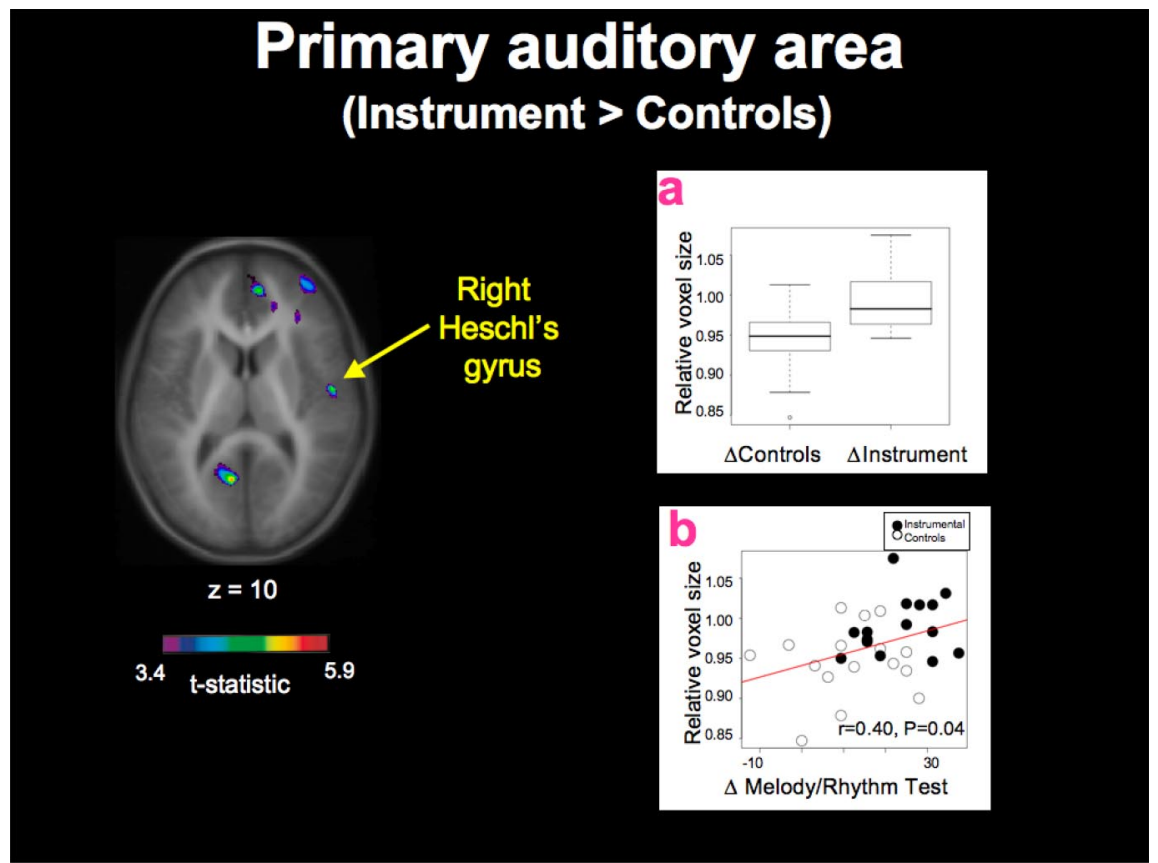

Figure 3. Longitudinal group brain deformation differences and brain-behavioral correlations in right primary auditory area. The brain image (a horizontal slice) shows areas of significant difference in relative voxel size over 15 months in instrumental ( $n=$ 15) versus control ( $n=16$ ) children in terms of a $t$-statistical color map of the significant clusters superimposed on an average MR image of all children ( $n=31$ ). The yellow arrow points to the right primary auditory region (lateral aspect of Heschl's gyrus). To illustrate the group differences, the relative voxel size is plotted for each group at the most significant (peak) voxel in the right primary auditory region ( $x=55, y=-8, z=10 ; t=4.9, p<0.1$ at a priori cluster threshold) (a). The significant positive correlations of relative voxel size with behavioral difference scores (from time 1 to time 2 ) of each child is shown for the melody/ rhythm test at the peak voxel in the right primary auditory area $(\boldsymbol{b})$. tween the brain deformation measures and the melody/rhythmic test battery in the right primary auditory region is consistent with functional brain mapping studies that have found activity changes using auditory-musical tests in similar auditory regions (Zatorre et al., 2002).

While structural brain differences were expected in motor and auditory brain areas, unexpected significant brain deformation differences were also found in various frontal areas, the left posterior pericingulate, and a left middle occipital region. However, none of these unexpected deformation changes were correlated with motor or auditory test performance changes. While we do not currently have an interpretation for some of these unexpected brain findings since they did not correlate with the auditory and motor behaviors, the left posterior pericingulate region warrants additional discussion since it showed a highly significant deformation difference. This region lies in the vicinity of Brodmann area 31 in the transition between posterior cingulate and occipital cortex and is involved in the integration of sensory (mostly visual) information and the limbic system. Such integration is involved in learning to read musical notation and relating music to its emotional content. The relative voxel size increases in frontomesial regions also stand out, although no obvious relationship with changes in motor and auditory performance was seen in these regions. Overall, these findings indicate that plasticity can occur in brain regions that control primary functions important for playing a musical instrument, and also in brain regions that might be responsible for the kind of multimodal sensorimotor integration likely to underlie the instrumental learning. None of the unexpected brain deformation differences mentioned above were correlated with behavioral performance changes in any of the far-transfer domains. This may indicate that brain structural changes in association areas and multimodal integration regions may develop before the emergence of significant behavioral/cognitive changes in fartransfer domains.

While we have discussed the functional significance of the brain-behavioral structural changes, the underlying structural properties of the results are not trivial to explain. The brain deformation techniques used here are key to localize brain size/shape changes over time, but are not able to inform us on the microstructural nature of these changes. Overall, instrumental children showed greater relative 
voxel size expansion than controls over the 15 months, and only one area of voxel size contraction. A voxel expansion or contraction may reflect increased or decreased gray or white matter due to neural reorganization/pruning or increased/decreased brain connectivity. Evidence from animal models investigating the effects of long-term learning and practice of complex motor skills (Anderson et al., 2002) on brain structure may shed light on the structural neural basis of the brain structural changes seen here. Several groups have demonstrated microstructural brain changes as a function of long-term motor learning, including an increased number of synapses and glial cells, increased density of capillaries in primary motor cortex and cerebellum, and new brain cells in the hippocampus after long-term motor training in adult rats (Black et al., 1990; Isaacs et al., 1992; Anderson et al., 1994; Kleim et al., 1996; Kempermann et al., 1997; Anderson et al., 2002). The sum of these microstructural changes could amount to structural differences that are detectable on a macrostructural level, such as those observed in the present study (Anderson et al., 2002; Bangert and Schlaug, 2006). It is possible that the specific and continuous engagement of a unimodal and multimodal sensorimotor network, and the induced changes in this network across a musician's career, may provide the neural basis for some of the sensorimotor and cognitive enhancements attributed to musical training. Future, even higher-resolution morphometric investigations with more direct measures of gray and white matter will be key to developing a better understanding of the underlying nature of the brain deformation differences found here. We also did not find any differences in MR intensities between groups, though using T1-weighted sequences is clearly a limitation in this regard. Future studies should examine quantitative sequences, such as diffusion tensor imaging, magnetization transfer, etc., in more detail to see whether microstructural changes can be captured separately from the volumetric differences described herein. Last, we wish to point out that one of the potential confounds of deformation-based morphometry is that the deformation procedure can sometimes result in changes being propagated to regions distant from their actual origin. Given that the present results were predicted based on the functional literature, we feel it is unlikely that such propagation accounts for the results presented in this manuscript. In the future, converging results from additional structural and functional analyses metrics will serve to strengthen our conclusions.

In summary, our findings show for the first time that musical training over only 15 months in early childhood leads to structural brain changes that diverge from typical brain development. Regional training-induced structural brain changes were found in musically relevant regions that were driven by musically relevant behavioral tests. The fact there were no structural brain differences found between groups before the onset of musical training indicates that the differential development of these brain regions is induced by instrumental practice rather by than preexisting biological predictors of musicality. These results provide new evidence for training-induced structural brain plasticity in early childhood. These findings of structural plasticity in the young brain suggest that long-term intervention programs can facilitate neuroplasticity in children. Such an intervention could be of particular relevance to children with developmental disorders and to adults with neurological diseases.

\section{References}

Anderson BJ, Li X, Alcantara AA, Isaacs KR, Black JE, Greenough WT (1994) Glial hypertrophy is associated with synaptogenesis following motor-skill learning, but not with angiogenesis following exercise. Glia 11:73-80.
Anderson BJ, Eckburg PB, Relucio KI (2002) Alterations in the thickness of motor cortical subregions after motor-skill learning and exercise. Learn Mem 9:1-9.

Bangert M, Schlaug G (2006) Specialization of the specialized in features of external human brain morphology. Eur J Neurosci 24:1832-1834.

Bangerter A, Heath C (2004) The Mozart effect: tracking the evolution of a scientific legend. Br J Soc Psychol 43:605-623.

Bermudez P, Zatorre RJ (2005) Differences in gray matter between musicians and nonmusicians. Ann N Y Acad Sci 1060:395-399.

Black JE, Isaacs KR, Anderson BJ, Alcantara AA, Greenough WT (1990) Learning causes synaptogenesis, whereas motor activity causes angiogenesis, in cerebellar cortex of adult rats. Proc Natl Acad Sci U S A 87:5568-5572.

Chan AS, Ho YC, Cheung MC (1998) Music training improves verbal memory. Nature 396:128.

Collins DL, Neelin P, Peters TM, Evans AC (1994) Automatic 3D intersubject registration of MR volumetric data in standardized Talairach space. J Comput Assist Tomogr 18:192-205.

Draganski B, May A (2008) Training-induced structural changes in the adult human brain. Behav Brain Res 192:137-142.

Draganski B, Gaser C, Busch V, Schuierer G, Bogdahn U, May A (2004) Neuroplasticity: changes in grey matter induced by training. Nature 427:311-312.

Elbert T, Pantev C, Wienbruch C, Rockstroh B, Taub E (1995) Increased cortical representation of the fingers of the left hand in string players. Science 270:305-307.

Forgeard M, Winner E, Norton A, Schlaug G (2008) Practicing a musical instrument in childhood is associated with enhanced verbal ability and nonverbal reasoning. PLoS One 3:e3566.

Friston KJ, Worsley KJ, Frackowiak RSJ, Mazziotta JC, Evans AC (1994) Assessing the significance of focal activations using their spatial extent. Hum Brain Mapp 1:210-220.

Fujioka T, Ross B, Kakigi R, Pantev C, Trainor LJ (2006) One year of musical training affects development of auditory cortical-evoked fields in young children. Brain 129:2593-2608.

Gaab N, Schlaug G (2003) Musicians differ from nonmusicians in brain activation despite performance matching. Ann NY Acad Sci 999:385-388.

Gaser C, Schlaug G (2003a) Brain structures differ between musicians and non-musicians. J Neurosci 23:9240-9245.

Gaser C, Schlaug G (2003b) Gray matter differences between musicians and nonmusicians. Ann N Y Acad Sci 999:514-517.

Genovese CR, Lazar NA, Nichols T (2002) Thresholding of statistical maps in functional neuroimaging using the false discovery rate. Neuroimage 15:870-878.

Grodd W, Hülsmann E, Lotze M, Wildgruber D, Erb M (2001) Sensorimotor mapping of the human cerebellum: FMRI evidence of somatotopic organization. Hum Brain Mapp 13:55-73.

Ho YC, Cheung MC, Chan AS (2003) Music training improves verbal but not visual memory: cross-sectional and longitudinal explorations in children. Neuropsychology 17:439-450.

Hund-Georgiadis M, von Cramon DY (1999) Motor-learning-related changes in piano players and non-musicians revealed by functional magnetic-resonance signals. Exp Brain Res 125:417-425.

Isaacs KR, Anderson BJ, Alcantara AA, Black JE, Greenough WT (1992) Exercise and the brain: angiogenesis in the adult rat cerebellum after vigorous physical activity and motor skill learning. J Cereb Blood Flow Metab 12:110-119.

Karni A, Meyer G, Jezzard P, Adams MM, Turner R, Ungerleider LG (1995) Functional MRI evidence for adult motor cortex plasticity during motor skill learning. Nature 377:155-158.

Kempermann G, Kuhn HG, Gage FH (1997) More hippocampal neurons in adult mice living in an enriched environment. Nature 386:493-495.

Kleim JA, Lussnig E, Schwarz ER, Comery TA, Greenough WT (1996) Synaptogenesis and Fos expression in the motor cortex of the adult rat after motor skill learning. J Neurosci 16:4529-4535.

Koelsch S, Fritz T, Schulze K, Alsop D, Schlaug G (2005) Adults and children processing music: an fMRI study. Neuroimage 25:1068-1076.

Lappe C, Herholz SC, Trainor LJ, Pantev C (2008) Cortical plasticity induced by short-term unimodal and multimodal musical training. J Neurosci 28:9632-9639. 
Lee DJ, Chen Y, Schlaug G (2003) Corpus callosum: musician and gender effects. Neuroreport 14:205-209.

Lotze M, Scheler G, Tan HR, Braun C, Birbaumer N (2003) The musician's brain: functional imaging of amateurs and professionals during performance and imagery. Neuroimage 20:1817-1829.

Münte TF, Kohlmetz C, Nager W, Altenmüller E (2001) Neuroperception. Superior auditory spatial tuning in conductors. Nature 409:580.

Norton A, Winner E, Cronin K, Overy K, Lee DJ, Schlaug G (2005) Are there pre-existing neural, cognitive, or motoric markers for musical ability? Brain Cogn 59:124-134.

Overy K, Norton AC, Cronin KT, Gaab N, Alsop DC, Winner E, Schlaug G (2004) Imaging melody and rhythm processing in young children. Neuroreport 15:1723-1726.

Oztürk AH, Tasçioglu B, Aktekin M, Kurtoglu Z, Erden I (2002) Morphometric comparison of the human corpus callosum in professional musicians and non-musicians by using in vivo magnetic resonance imaging. J Neuroradiol 29:29-34.

Pantev C, Oostenveld R, Engelien A, Ross B, Roberts LE, Hoke M (1998) Increased auditory cortical representation in musicians. Nature 392:811-814.

Rauscher FH, Shaw GL, Ky KN (1993) Music and spatial task performance. Nature 365:611.

Rauscher FH, Shaw GL, Levine LJ, Wright EL, Dennis WR, Newcomb RL (1997) Music training causes long-term enhancement of preschool children's spatial-temporal reasoning. Neurol Res 19:2-8.

Rauscher FH, Robinson KD, Jens JJ (1998) Improved maze learning through early music exposure in rats. Neurol Res 20:427-432.

Raven JC (1976a) Coloured progressive matrices. Oxford: Oxford Psychologists.

Raven JC (1976b) Standard progressive matrices. Oxford: Oxford Psychologists.

Robbins S, Evans AC, Collins DL, Whitesides S (2004) Tuning and comparing spatial normalization methods. Med Image Anal 8:311-323.

Rosner J, Simon D (1971) Test of auditory analysis skill, TAAS. J Learn Disabil 4:40-48.

Schellenberg EG (2004) Music lessons enhance IQ. Psychol Sci 15:511-514.

Schlaug G (2001) The brain of musicians. A model for functional and structural adaptation. Ann N Y Acad Sci 930:281-299.

Schlaug G, Jäncke L, Huang Y, Staiger JF, Steinmetz H (1995) Increased corpus callosum size in musicians. Neuropsychologia 33:1047-1055.

Schlaug G, Norton A, Overy K, Winner E (2005) Effects of music training on the child's brain and cognitive development. Ann N Y Acad Sci 1060:219-230.

Schmithorst VJ, Wilke M (2002) Differences in white matter architecture between musicians and non-musicians: a diffusion tensor imaging study. Neurosci Lett 321:57-60.

Schneider P, Scherg M, Dosch HG, Specht HJ, Gutschalk A, Rupp A (2002) Morphology of Heschl's gyrus reflects enhanced activation in the auditory cortex of musicians. Nat Neurosci 5:688-694.

Shahin AJ, Roberts LE, Chau W, Trainor LJ, Miller LM (2008) Music training leads to the development of timbre-specific gamma band activity. Neuroimage 41:113-122.

Sled JG, Zijdenbos AP, Evans AC (1998) A nonparametric method for automatic correction of intensity nonuniformity in MRI data. IEEE Trans Med Imaging 17:87-97.

Sluming V, Barrick T, Howard M, Cezayirli E, Mayes A, Roberts N (2002) Voxel-based morphometry reveals increased gray matter density in Broca's area in male symphony orchestra musicians. Neuroimage 17:1613-1622.

Sluming V, Brooks J, Howard M, Downes JJ, Roberts N (2007) Broca's area supports enhanced visuospatial cognition in orchestral musicians. J Neurosci 27:3799-3806.

Sowell ER, Thompson PM, Leonard CM, Welcome SE, Kan E, Toga AW (2004) Longitudinal mapping of cortical thickness and brain growth in normal children. J Neurosci 24:8223-8231.

Steele KM, Brown JD, Stoecker JA (1999) Failure to confirm the Rauscher and Shaw description of recovery of the Mozart effect. Percept Mot Skills $88: 843-848$.

Vaughn K (2000) Music and mathematics: modest support for the oftclaimed relationship. J Aesthet Educ 34:149-166.

Wahl M, Lauterbach-Soon B, Hattingen E, Jung P, Singer O, Volz S, Klein JC, Steinmetz H, Ziemann U (2007) Human motor corpus callosum: topography, somatotopy, and link between microstructure and function. J Neurosci 27:12132-12138.

Wechsler D (1991) WISC-III Wechsler Intelligence Scale for Childrenthird edition: manual. San Antonio, TX: The Psychological Corporation.

Witelson SF (1989) Hand and sex differences in the isthmus and genu of the human corpus callosum. A postmortem morphological study. Brain 112:799-835.

Worsley KJ, Taylor JE, Tomaiuolo F, Lerch J (2004) Unified univariate and multivariate random field theory. Neuroimage 23[Suppl 1]:S189-S195.

Zatorre RJ (1998) Functional specialization of human auditory cortex for musical processing. Brain 121:1817-1818.

Zatorre RJ, Belin P, Penhune VB (2002) Structure and function of auditory cortex: music and speech. Trends Cogn Sci 6:37-46.

Zatorre RJ, Chen JL, Penhune VB (2007) When the brain plays music: auditory-motor interactions in music perception and production. Nat Rev Neurosci 8:547-558. 